\title{
Renal Safety of Hydroxyethyl starch 130/0.42 After Cardiac Surgery: A Retrospective Cohort Analysis
}

\author{
Benedict Morath ${ }^{1,2,3} \cdot$ Andreas D. Meid ${ }^{1} \cdot$ Johannes Rickmann ${ }^{4} \cdot J^{\prime}$ asmin Soethoff ${ }^{5}$ Markus Verch ${ }^{5} \cdot$ Matthias Karck $^{5}$. \\ Marcin Zaradzki ${ }^{5}$
}

Accepted: 29 August 2021 / Published online: 26 September 2021

(c) The Author(s) 2021

\begin{abstract}
Introduction The risk for renal complications from hydroxyethyl starch 130/0.42 (HES) impacts treatment decisions in patients after cardiac surgery.

Objective The objective of this study was to determine the impact of postoperatively administered HES on renal function and 90-day mortality compared to sole crystalloid administration in patients after elective cardiac surgery.

Methods Using electronic health records from a university hospital, confounding-adjusted models analyzed the associations between postoperative HES administration and the occurrence of postoperative acute kidney injury. In addition, 90-day mortality was evaluated. The impact of HES dosage and timing on renal function on trajectories of estimated glomerular filtration rates over the postoperative period was investigated using linear mixed-effects models.

Results Overall 1009 patients (45.0\%) experienced acute kidney injury. Less acute kidney injury occurred in patients receiving HES compared with patients receiving only crystalloids for fluid resuscitation $(43.7 \%$ vs $51.2 \%, p=0.008)$. In multivariate acute kidney injury models, HES had a protective association (odds ratio: $0.89 ; 95 \%$ confidence interval $0.82-0.96$ ). Crystalloids were not as protective as HES (odds ratio: 0.98; 95\% confidence interval 0.95-1.00). There was no association between HES and 90-day mortality (odds ratio: 1.05; 95\% confidence interval 0.88-1.25). Renal function trajectories were dose dependent and biphasic, HES appeared to slow down the late postoperative decline.

Conclusions This study showed no association between HES and the postoperative occurrence of acute kidney injury and thus further closes the evidence gap on HES safety in cardiac surgery patients. Although this was a retrospective cohort study, the results indicated that HES might be safely administered to cardiac surgery patients with regard to renal outcomes, especially if it was administered early and dosed appropriately.
\end{abstract}

Benedict Morath and Andreas D. Meid contributed equally and should be considered joint first author.

Marcin Zaradzki

marcin.zaradzki@med.uni-heidelberg.de

1 Department of Clinical Pharmacology and Pharmacoepidemiology, Heidelberg University Hospital, Heidelberg, Germany

2 Cooperation Unit Clinical Pharmacy, Heidelberg University, Heidelberg, Germany

3 Hospital Pharmacy, Heidelberg University Hospital, Heidelberg, Germany

4 Center for Information and Medical Technology, Heidelberg University Hospital, Heidelberg, Germany

5 Department of Cardiac Surgery, University Hospital Heidelberg, Heidelberg, Germany 


\section{Key Points}

In this retrospective analysis, postoperative administration of hydroxyethyl starch 130/0.42 did not increase the risk for acute kidney injury or 90-day mortality in patients after elective coronary artery bypass grafting, aortic valve replacement, or a combination of both.

Early administration of hydroxyethyl starch 130/0.42 in a low dosage showed the potential to slow down the postoperative decline of the renal function after cardiac surgery.

When transferring and extrapolating renal safety data, patient populations and clinical settings might be of particular importance and might modulate the effects.

\section{Introduction}

After weaning patients from cardiopulmonary bypass (CPB), it is essential to stabilize hemodynamics with intravenous fluids [1-3]. Among the possible therapeutic options, hydroxyethyl starch (HES) has favorable hemodynamic properties (e.g., long intravascular half-life); it is used postoperatively especially for fluid resuscitation when crystalloid solutions are not effective enough. Several prospective trials in other settings have reported adverse outcomes for different HES products [1, 4-7]. However, these trials, conducted in patients experiencing septic shock or similar conditions, concluded HES can induce severe renal failure, bleeding complications, and increase mortality compared with crystalloids alone [8-10]. Consequently, the European Medicines Agency considered the market withdrawal and eventually restricted HES administration to patients with hemorrhagic hypovolemia [11]. The debate on the benefit that HES spurred was additionally affected by issues beyond science, such as litigation, research misconduct, and even criminality [12-16].

However, from a research perspective, these points should not preclude a scientific debate and research on HES safety in special patient cohorts and settings. Although there are no prospective trials investigating HES safety in cardiac surgery patients, HES is still used in concordance with current guidelines. There is an unmet and urgent need for this evidence in the cardiac surgery realm, especially in view of recent results of HES administration in abdominal surgery patients [17].

In a pharmacoepidemiologic approach using data from electronic health records, we performed a confounding-adjusted retrospective cohort analysis of consecutive patients undergoing elective cardiac surgery. The incidence of postoperative acute kidney injury (AKI) and 90-day mortality were investigated with respect to the amount and timing of postoperative HES fluid resuscitation.

\section{Methods}

\subsection{Source of Data}

All data were extracted from different sources within the hospital digital infrastructure. Data from the intensive care unit (ICU) [e.g., prescribed medication, dosages and timing of administration, or urine output] were extracted from the electronic patient charts (COPRA $\left.{ }^{\circledR}, \mathrm{COPRA} \mathrm{GmbH}\right)$. Moreover, data on sociodemographics, comorbidities, surgery process parameters (e.g., duration of surgery), and follow-up data (e.g., 90-day mortality) were taken from the electronic health record (i.s.h.-med ${ }^{\circledR}$, SAP). Finally, all laboratory parameters were extracted from the laboratory software (LAURIS ${ }^{\circledR}$, nexuslswisslab). All parameters and laboratory values were measured during the clinical routine, at the bedside, or within the in-house laboratory. For longitudinal variables extracted at specific time points or time intervals, the time of a patient's admission to the ICU was defined as time zero (Table S1 in the Electronic Supplementary Material [ESM]).

\subsection{Study Design}

In our main analysis, a retrospective cohort study was performed to investigate how early postoperative administration of HES 130/0.42 affects adverse outcomes in elective patients undergoing coronary artery bypass grafting, elective aortic valve replacement, or a combination of both. Furthermore, a sensitivity analysis was applied to a retrospective, propensity score-matched cohort design.

The primary study endpoint was to determine the impact of postoperatively administered HES on the incidence of postoperative AKI, as defined by the Kidney Disease Improving Global Outcomes (KDIGO) criteria. Secondary endpoints were mortality within 90 days of follow-up and to analyze how renal function changes over time depending on the HES dosage and administration times. The 'strengthening the reporting of observational studies in epidemiology' (STROBE) statement was used and the checklist is provided (Table S2 in the ESM) [18].

\subsection{Study Cohort}

The study cohort included all patients aged $>18$ years undergoing elective coronary artery bypass grafting, aortic 
valve replacement, or combined coronary artery bypass grafting plus aortic valve replacement surgery, for whom an electronic health record was available. The recruiting period was from March 2015 to January 2019 (before the restricted market access program). Patients undergoing emergency surgery, aged $<18$ years, or patients with planned cardiac surgeries other than the aforementioned procedures were not considered eligible for analysis and therefore excluded. Balanced crystalloids (i.e., ringer, ringer plus lactate) are usually preferred both intraoperatively and postoperatively. During surgery, the fluids commonly used are blood products and crystalloids; mostly no albumin is used during surgery. After surgery and at admission to the ICU, different goals as surrogates for volume resuscitation are achieved: mean arterial pressure $>65 \mathrm{mmHg}$, central venous pressure $\sim 10 \mathrm{mmHg}$, adequate stroke volume $(70 \mathrm{~mL})$, cardiac index $>2.1 \mathrm{l} /$ $\mathrm{min} / \mathrm{m}^{2}$, hemoglobin $\sim 10 \mathrm{~g} / \mathrm{dL}$, mixed venous saturation $>65 \%$, and noradrenaline not exceeding $0.05 \mathrm{mg} /$ $\mathrm{kg} / \mathrm{kg}$. Intraoperative transfusion triggers are according to international standards: hemoglobin $<8 \mathrm{~g} / \mathrm{dL}$ and hematocrit $<0.241 / 1$ in non-bleeding patients $[19,20]$. In patients with massive bleeding, transfusion is guided by fluid resuscitation principles and is usually in equal parts erythrocyte concentrate:fresh frozen plasma:thrombocyte concentrate in a ratio of $1: 1: 1$. The transfusion of other blood products is guided mostly by a rotational thromboelastometry assessment to reduce unnecessary transfusions [21]. For initial fluid substitution, balanced crystalloids are used but if patients do not respond adequately (e.g., lactate levels rise, mean arterial pressure decreases under $65 \mathrm{mmHg}$, central venous saturation falls below $60 \%$, or vasopressor administration needs to be intensified), HES might be administered in the ICU according to the attending physician's choice. As there was no standardized protocol for fluid therapy, the intensivist defines the therapy according to the previously described surrogates and triggers for fluid therapy.

\subsection{Study Outcomes}

In two cross-sectional models, the associations between HES administration with occurrence of postoperative AKI (as defined by the KDIGO criteria) and with 90-day mortality were investigated [22]. Applying these criteria to derive AKI stages in the postoperative follow-up, averages of serum creatinine levels within 12-h time intervals and cumulative urine output measurements in 6-h time intervals were obtained during the ICU stay. Longitudinal trajectories of renal function status were based on the postoperative estimated glomerular filtration rate (eGFR) and calculated in 12-h intervals with the formula introduced by the Chronic Kidney Disease Epidemiology Collaboration [23].

\subsection{Exposure}

Exposure to HES was available as the absolute amount in milliliters, representing a continuous variable. Based on this, different operationalizations were considered for the analyses: overall administration (yes/no), dosage $(\mathrm{mL})$, and timing of administration. Total cumulative dosages were calculated and thereafter divided into categories: low $(<1000 \mathrm{~mL})$, middle (1000-2000 mL), and high $(>2000 \mathrm{~mL})$. Timing of administration was also split into the following categories: early ( $<24 \mathrm{~h}$ postoperative), middle $(24-72 \mathrm{~h}$ postoperative), and late ( $>72 \mathrm{~h}$ postoperative). As there was no standardized protocol for volume therapy, the intensivist was responsible for the chosen therapy, in accordance with the current clinical situation.

\subsection{Potential Confounders}

Co-variates at the patient level (operationalization shown in parentheses, see also Table S1 in the ESM) were age and sex (male/female). Included comorbidities were considered as binary indicators, i.e., diabetes mellitus as type 1 or 2 diabetes including diabetes treated with dietary measures, and atrial fibrillation consisting of intermittent and persistent atrial fibrillation. Left ventricular ejection fraction was available at pre-defined categories in the electronic health record (left ventricular ejection fraction $>50 \%, 31-50 \%, 21-30 \%$, and $\leq 20 \%$ ). The logistic EuroSCORE was included as a measure of mortality risk [24]. The body mass index was calculated using the patients' admission weight and height.

With respect to surgery, the following parameters were considered: aortic cross clamp time, (time from the clamping start to release of the aorta [minutes]), surgery time (from skin incision until suture [minutes]), CPB time (from start to wean [minutes]), and cumulative intraoperative volume of blood transfusion $(\mathrm{mL})$ represented all packed red blood cells transfused during surgery. With regard to the ICU stay, ventilation time was defined as the total duration the patient was ventilated (minutes), the postoperatively administered platelet and packed red blood cells were available as the total amount $(\mathrm{mL})$, and drain losses as the cumulative volume lost over indwelling drains $(\mathrm{mL})$. Pre-operative serum creatinine $(\mathrm{mg} / \mathrm{dL})$ was measured at hospital admission. Postoperatively, serum creatinine $(\mathrm{mg} / \mathrm{dL})$ and blood urea $(\mathrm{mg} /$ $\mathrm{dL}$ ) were extracted in 12-hour intervals over the hospital stay. Hemodynamic parameters were mean arterial pressure $(\mathrm{mmHg})$ and systolic arterial blood pressure $(\mathrm{mmHg})$ in 12-h time intervals as measured at the bedside and reported in the electronic patient chart. 
In terms of medication and fluid management, the administration of crystalloids was available as cumulative volume $(\mathrm{mL})$ and based thereupon also as a binary (yes/no) indicator. Drugs with a reported nephrotoxic potential (vancomycin, gentamicin, tobramycin, amikacin, piperacillin/tazobactam, colistin, cotrimoxazol, lithium, acyclovir, tacrolimus, ciclosporin, etoricoxib, diclofenac, and ibuprofen) were grouped into the binary indicator named 'nephrotoxic drugs' [25-27]. Likewise, the administration of diuretics (e.g., mannitol, furosemide) and catecholamines (noradrenaline, adrenaline, dobutamine) was considered as binary indicator variables.

\subsection{Missing Data}

In total, 58 values were missing in five of the considered variables for modeling. This missing data pattern was considered as acceptable to avoid imputation [28]. Laboratory values were complete, to the extent that all measured values were available.

\subsection{Statistical Methods}

The retrospective cohort was described by statistics summarizing their distributional location parameters or their proportions, in the case of either continuous or categorical variables, respectively. Inferential tests providing descriptive $p$ values were chosen according to the variable scale and variable distribution.

Cross-sectional models were based on multivariate logistic regression models. In order to evaluate the robustness of a chosen approach, we additionally estimated odds ratios (ORs) for AKI with HES treatment in sensitivity analyses applying weighted and matched propensity score adjustment (Tables S3 and S4 in the ESM). Acknowledging that the decision for HES treatment is not regularly a binary choice, but rather a question of dose, timing, and combination with crystalloids (Fig. S1 in the ESM), we chose multivariate models comprehensively adjusted for confounding as the main analysis. Covariates in logistic regression models were empirically determined by the preceding variable selection based on the 'Least Absolute Shrinkage and Selection Operator' (LASSO) [29-31]. The mandatory variables of age, pre-operative serum creatinine, administration of HES, and crystalloid solutions were left unregularized and thus included in any model. Further candidate predictors were logistic EuroSCORE, left ventricular function, atrial fibrillation, diabetes, administration of catecholamines, nephrotoxic drugs, furosemide, mannitol, drain losses, mean arterial pressure, systolic pressure, intraoperative blood substitution, postoperative blood substitution, and duration of surgery. Exploring potential non-linear relationships with administered amounts of HES, generalized additive models were fitted with spline functions for HES with linear adjustment for the remaining covariates of the structural model [32].

Longitudinal models for eGFR trajectories over time were based on linear mixed-effects models [33]. A random intercept for the patient and a random slope for time accounted for intra-individual variability. The influence of HES administration in time intervals of $24 \mathrm{~h}$ was modeled as an interaction term between cumulative HES administration and time. The 'nlimb' optimization algorithm was applied to achieve well-converging models with a set of clinically justified covariates for confounding adjustment $[31,34]$. Inferential tests on parameter estimates were based on t-tests using Satterthwaite's method [35]. In general, statistical tests were two-sided, and $95 \%$ confidence intervals were calculated according to an alpha level of 0.05 . All analyses were conducted using the R software environment in version 3.6.1 (R Foundation for Statistical Computing, Vienna, Austria).

\section{Results}

\subsection{Participants}

Overall, 2245 patients were screened and all 2245 patients were included in the analysis. Of these, 371 patients (16.5\%) received only balanced crystalloids for fluid resuscitation and 1874 patients $(83.5 \%)$ received a combination of balanced crystalloids and HES early after ICU admission. The baseline characteristics showed minor differences (Table 1). As expected, patients in the HES group had a slightly better renal function at ICU admission. Detailed information on vasopressor therapy and volume status surrogates in the ICU is available in the ESM (Figs. S2 and S3).

All patients received on average $3202 \pm 1291 \mathrm{~mL}$ of crystalloids in the first postoperative $12 \mathrm{~h}$ in the ICU, excluding crystalloids administered during surgery. Additional data on the association between the dose of administered crystalloids and HES, as well as the overall timing of the administration of crystalloids and HES, are available (Fig. S1 in the ESM). Patients who thereafter did not show adequate volume resuscitation with crystalloids after significant blood loss during surgery $(n=1874)$ received on average $1091 \pm 649 \mathrm{~mL}$ of HES (Table S5 in the ESM). Bleedings and reoperations were numerically higher in the HES cohort (3.2\% and 3.4\% compared with $2.1 \%$ and $1.1 \%$ in the nonHES group, respectively), but no association between the amount of administered HES and these events was found after multivariate adjustment (Table S6 in the ESM). Acute kidney injury (any KDIGO stage) and these events 
Table 1 Baseline patient characteristics at admission to the intensive care unit (ICU) and during the ICU stay

\begin{tabular}{|c|c|c|c|}
\hline Characteristics & No HES $(n=371)$ & $\operatorname{HES}(n=1874)$ & $P$ value \\
\hline Age (years) & $69.7 \pm 10.3$ & $69.0 \pm 9.6$ & 0.216 \\
\hline Male, $n(\%)$ & $269(72.5)$ & $1449(77.3)$ & 0.046 \\
\hline Atrial fibrillation, $n(\%)$ & & & $<0.001$ \\
\hline Intermittent & $56(15.1)$ & 185 (9.9) & \\
\hline Persistent & $29(7.8)$ & $88(4.7)$ & \\
\hline Chronic & $9(2.43)$ & $49(2.62)$ & \\
\hline Current or previous smoking, $n(\%)$ & $154(41.5)$ & $783(42.8)$ & 0.922 \\
\hline Diabetes mellitus, $n(\%)$ & $141(38.0)$ & $645(34.4)$ & 0.186 \\
\hline Hypertension, $n(\%)$ & $336(90.6)$ & 1665 (88.9) & 0.332 \\
\hline Logistic EUROscore & $7.1 \pm 3.6$ & $6.4 \pm 3.1$ & $<0.001$ \\
\hline LVEF, $n(\%)$ & & & 0.058 \\
\hline LVEF $>50 \%$ & $225(60.7)$ & $1241(66.2)$ & \\
\hline LVEF $31-50 \%$ & $115(31.0)$ & $510(27.2)$ & \\
\hline LVEF $21-30 \%$ & $25(6.7)$ & $110(5.9)$ & \\
\hline LVEF $<20 \%$ & $6(1.6)$ & $10(0.5)$ & \\
\hline Systolic arterial pressure $(\mathrm{mmHg})$ & $155 \pm 31$ & $158 \pm 32$ & 0.069 \\
\hline $\mathrm{PAD}, n(\%)$ & $66(17.8)$ & $240(12.8)$ & 0.011 \\
\hline \multicolumn{4}{|l|}{ Laboratory } \\
\hline eGFR at ICU admission $\left(\mathrm{mL} / \mathrm{min} / 1.73 \mathrm{~m}^{2}\right)$ & $69.3 \pm 27.2$ & $80.3 \pm 19.8$ & $<0.001$ \\
\hline Hemoglobin $(g / d L)$ & $11.2 \pm 1.1$ & $11.0 \pm 1.1$ & 0.006 \\
\hline Lactate $(\mathrm{mg} / \mathrm{dL})$ & $15.7 \pm 11.7$ & $15.8 \pm 9.3$ & 0.874 \\
\hline Leucocytes $(/ \mathrm{nl})$ & $12.2 \pm 5.2$ & $10.8 \pm 3.7$ & $<0.001$ \\
\hline Maximum creatinine in ICU (mg/dL) & $1.39 \pm 1.42$ & $1.03 \pm 0.71$ & $<0.001$ \\
\hline Preoperative creatinine (mg/dL) & $1.09 \pm 0.5$ & $0.95 \pm 0.33$ & $<0.001$ \\
\hline Thrombocytes $(/ \mathrm{nl})$ & $178 \pm 64$ & $163 \pm 60$ & $<0.001$ \\
\hline Urea (mg/dL) & $49 \pm 30$ & $39 \pm 19$ & $<0.001$ \\
\hline \multicolumn{4}{|l|}{ Surgical parameters } \\
\hline Aortic clamp time (min) & $45 \pm 32$ & $58 \pm 26$ & $<0.001$ \\
\hline Bypass time (min) & $73 \pm 50$ & $96 \pm 45$ & $<0.001$ \\
\hline Surgery time (min) & $198 \pm 65$ & $224 \pm 63$ & $<0.001$ \\
\hline Total blood substitution (mL) & $321 \pm 478$ & $362 \pm 504$ & 0.144 \\
\hline Total FFP substitution (mL) & $14 \pm 125$ & $22 \pm 152$ & 0.341 \\
\hline Total thrombocyte substitution (mL) & $54 \pm 214$ & $80 \pm 280$ & 0.091 \\
\hline \multicolumn{4}{|l|}{ ICU parameters/medication } \\
\hline Ventilation time (min) & $988 \pm 1231$ & $1553 \pm 3241$ & $<0.001$ \\
\hline Catecholamine yes, $n(\%)$ & $237(63.9)$ & $1455(77.6)$ & $<0.001$ \\
\hline Total dose of crystalloids (mL) & $5093 \pm 1940$ & $6586 \pm 2381$ & $<0.001$ \\
\hline Nephrotoxic drugs yes, $n(\%)$ & $26(7.0)$ & $159(8.5)$ & 0.345 \\
\hline Mannitol yes, $n(\%)$ & $2(0.5)$ & $5(0.3)$ & 0.399 \\
\hline Total blood substitution (mL) & $239 \pm 552$ & $365 \pm 755$ & 0.002 \\
\hline Total FFP substitution (mL) & $33 \pm 330$ & $49 \pm 387$ & 0.462 \\
\hline Total thrombocyte substitution (mL) & $58 \pm 236$ & $85 \pm$ & 0.076 \\
\hline
\end{tabular}

The $p$-values relate to the unbalanced nature of both groups and describe the extent to which a tabulated characteristic was associated with allocation to one group or the other

eGFR estimated glomerular filtration rate, FFP fresh frozen plasma, HES hydroxyethyl starch 130/0.42, $L V E F$ left ventricular ejection fraction, min minutes, $P A D$ peripheral arterial disease 
Table 2 Number of patients who experienced renal failure after surgery or died during the follow-up

\begin{tabular}{lcll}
\hline Endpoint & No HES & HES & $P$ value \\
\hline No AKI, $n(\%)$ & $181(48.8)$ & $1055(56.3)$ & $\begin{array}{l}\text { Reference for } \\
\text { each } 2 \times 2 \\
\text { table }\end{array}$ \\
KDIGO stage I, $n(\%)$ & $72(19.4)$ & $274(14.6)$ & 0.007 \\
KDIGO stage II, $n(\%)$ & $59(15.9)$ & $219(11.7)$ & 0.009 \\
KDIGO stage III, $n(\%)$ & $59(15.9)$ & $326(17.4)$ & 0.81 \\
KDIGO any stage, $n(\%)$ & $190(51.2)$ & $819(43.7)$ & 0.009 \\
Death up to day 90, $n(\%)$ & $14(3.8)$ & $63(3.4)$ & 0.691 \\
\hline
\end{tabular}

AKI acute kidney injury, HES hydroxyethyl starch 130/0.42, KDIGO Kidney Disease: Improving Global Outcomes

were not correlated and independent of each other (PChisquare $($ bleeding $)=1$, PChi-square $($ reoperation $)=0.111$ )

\subsection{Cross-Sectional AKI and Mortality Models}

Postoperatively, a total of 1009 patients (45.0\%) developed AKI. However, significantly less AKI of any KDIGO stage was noted in the HES group (43.7\%) compared with the crystalloid group $(51.2 \%$ ) (Table 2). In detail, the AKI occurrence mainly differed significantly at KDIGO stages I and II, whereas there was no difference at stage III. There was no difference in 90-day mortality between the groups $(3.4 \%$ vs $3.8 \%)$ (Table 2 ).

In the multivariate AKI model, the selected co-variates showed clinically expected associations with the occurrence of AKI (Fig. 1, Table S7 in the ESM). Particularly, fluid administration showed a marginally negative association per every $500 \mathrm{~mL}$ of administered crystalloid (OR 0.98 [0.95-1.00]) and a stronger effect per every $500 \mathrm{~mL}$ of HES (OR 0.89 [0.82-0.96]). The results were in line with the propensity score-adjusted sensitivity analysis (Table S4 in the ESM). Regarding timing of HES administration, administration within $<24 \mathrm{~h}$ after surgery was seen to have a significant negative association $(p=0.0277)$ that was not present for administration between 24 and $72 \mathrm{~h}$, and $>72 \mathrm{~h}$. In the co-variate-adjusted model for 90-day mortality, a borderline increased risk was observed for every additionally administered $500 \mathrm{~mL}$ of crystalloids (OR 1.06 [1.00-1.11]), while the administration of HES was not associated with mortality (OR 1.05 [0.88-1.25]) (Fig. 2; Table S8 in the ESM).

\subsection{Longitudinal eGFR Trajectories After HES Administration}

A linear mixed-effects model successfully described the impact of HES administration on the longitudinal eGFR

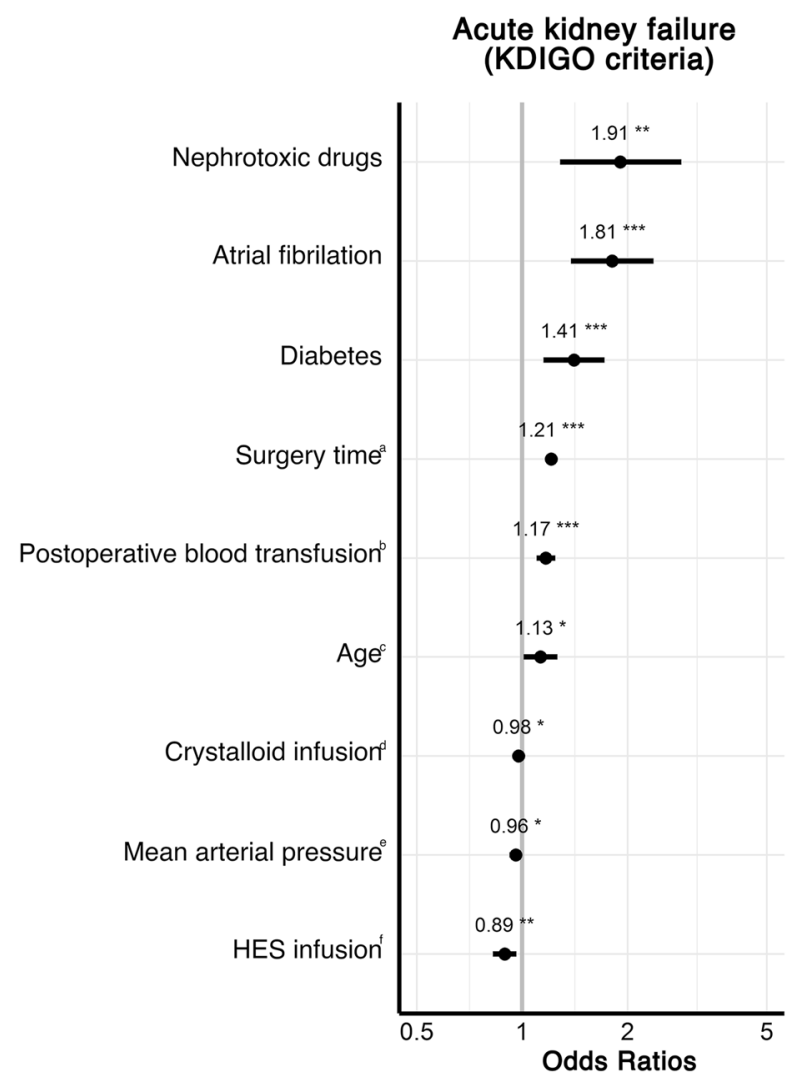

Abbreviations: HES: Hydroxyethyl starch I KDIGO: Kidney Disease Improving Global Outcomes Legend: a: Per 15 minutes I b: Per $300 \mathrm{~mL}$ I c: Per 10 years I d: Per $500 \mathrm{~mL}$ | e: Per $10 \mathrm{mmHg}$ |

If: Per $\left.500 \mathrm{~mL}\right|^{*}: p<\left.0.05\right|^{* *}: p<\left.0.01\right|^{* * *}: p<0.001$

Fig. 1 Association of hydroxyethyl starch administration by $500 \mathrm{~mL}$ with the occurrence of an acute kidney injury indicated by any Kidney Disease: Improving Global Outcomes (KDIGO) stage after surgery. Among all selected covariates (Table S7 of ESM), only those reaching statistical significance are shown $(* p<0.05, * * p<0.01$, $* * * p<0.001) .57156$ observations were deleted because of missingness

trajectory and allowed the prediction of trajectories for virtual patients. As such, we chose virtual patients with median values in the independent variables and different doses of HES at different administration times (Fig. 3; Table S9 in the ESM). Overall, renal function declined after cardiac surgery independent of the chosen fluid regimen. However, in contrast to the near linear decline after sole crystalloid administration, HES showed a biphasic decline pattern due to the significant interaction with time after surgery. This resulted in dose-dependent drops of eGFR estimates. The eGFR decline gradually slowed down in all HES-treated patients, and eGFR even slightly improved compared with patients receiving crystalloid therapy (Fig. 3). For further exploration, we provide an online calculator (https://hydro xyethylstarch-cardiacsurgery.shinyapps.io/shiny/). 


\section{0-day mortality}

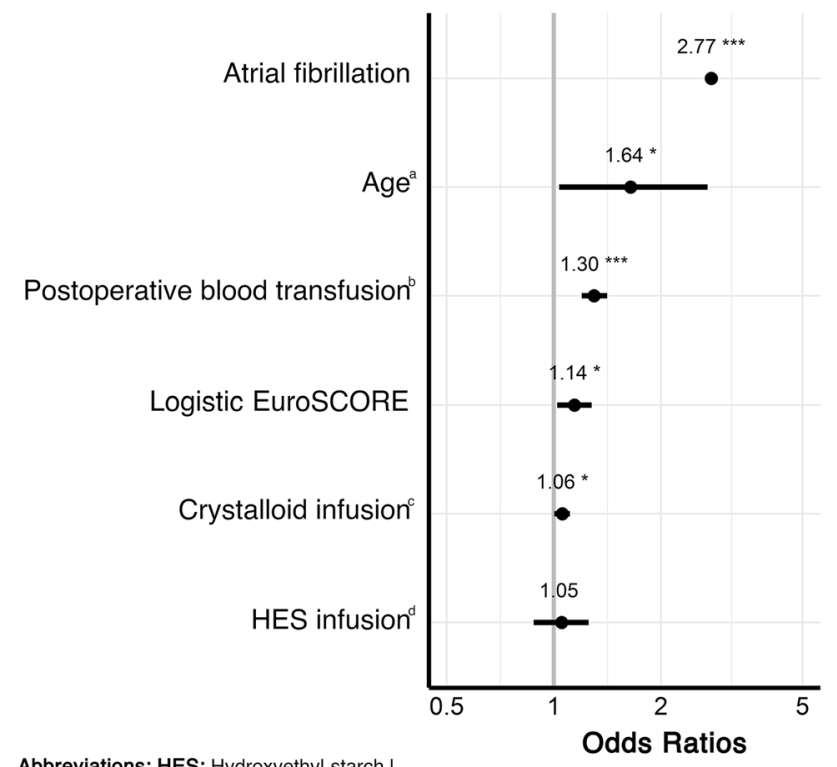

Abbreviations: HES: Hydroxyethyl starch I

Legend: a: Per 10 years | b: Per $300 \mathrm{~mL}$ । c: Per $500 \mathrm{~mL}$ । d: Per $500 \mathrm{~mL}$ ।

$\left.\right|^{*}: p<\left.0.05\right|^{* *}: p<\left.0.01\right|^{* * *}: p<0.001$

Fig. 2 Association of hydroxyethyl starch administration by $500 \mathrm{~mL}$ with death within 90 days of the follow-up after surgery. Among all selected covariates (Table S8 of the ESM), only those reaching statistical significance are shown $\left({ }^{*} p<0.05,{ }^{* *} p<0.01\right.$, *** $\left.p<0.001\right)$. 57156 observations were deleted because of missingness

\section{Discussion}

In this study evaluating consecutive data of a large realworld patient cohort that underwent open cardiac surgery, exhaustively adjusted analyses could not detect any negative effect of HES administration on kidney function and mortality. Moreover, unexpected significant 'protective' associations of (especially early and low-dosed) HES administration were present and also evident from modeled postoperative eGFR trajectories. Overall, the reported incidence of AKI after cardiac surgery is heterogenic and reported to be between 5 and $43 \%$ depending on the centers and used diagnostic criteria [36, 37]. The incidence of this study appears to be in the upper range. Patients who experienced bleeding or needed reoperation consequently needed higher amounts of fluids especially HES for hemodynamic stabilization. This explains the numerically higher number of patients experiencing bleeding or reoperation in the HES cohort. Moreover, we know that bleeding or reoperation results in a higher incidence of AKI [38]. Taking this together, the HES cohort might have had an even higher risk for AKI.

These main findings do not concur with the actual recommendations for administration of HES and should be regarded with caution [8-10]. However, with regard to this study, the question might be raised whether findings collected in septic and renally insufficient patients can be generally extrapolated to postoperative cardiac surgery patients [39]. Potentially, adverse outcomes after HES administration

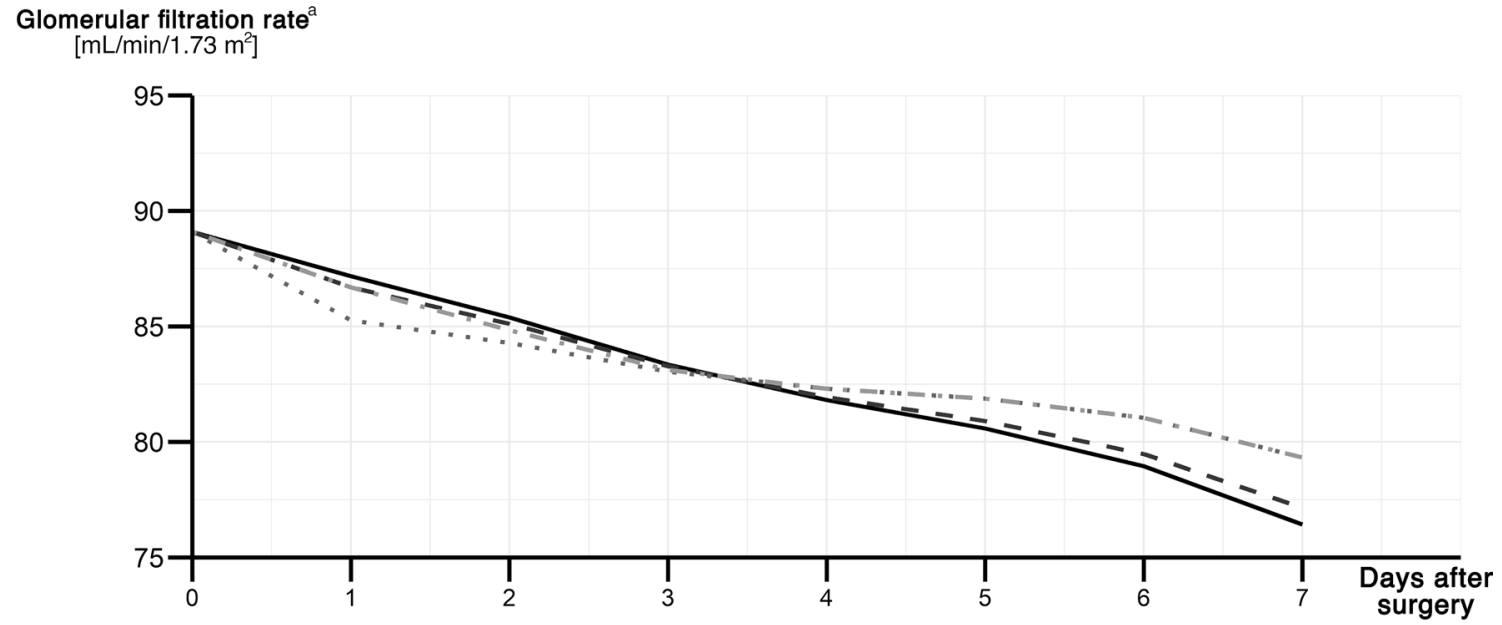

Legend: - No HES - $500 \mathrm{~mL}$ on day 1 " " $2000 \mathrm{~mL}$ on day 1 " = $500 \mathrm{~mL}$ on days 1 to 4

a: Defined by the Chronic Kidney Disease Epidemiology Collaboration (CKD-EPI) formula

Fig. 3 Predicted estimated glomerular filtration rate (eGFR) trajectory $\left(\mathrm{mL} / \mathrm{min} / 1.73 \mathrm{~m}^{2}\right)$ every $24 \mathrm{~h}$ after surgery for a situation of a patient with median values in each covariate (Table S9 of the ESM). Different administration schemes are projected in scenarios with no hydroxyethyl starch 130/0.42 (HES) [black solid line], $500 \mathrm{~mL}$ of HES on day 1 (dark gray dashed line), $2000 \mathrm{~mL}$ of HES on day 1 (medium gray dotted line), and each $500 \mathrm{~mL}$ of HES on days 1-4 (light gray dot-dashed line) 
differ between settings and patient populations, as do the physiologic processes in these patient populations.

After cardiac surgery, patients experience hemodynamic instability due to fluid shifts and a systemic inflammatory response syndrome triggered by the $\mathrm{CPB}$, resulting in endothelial leakage [40-43]. Consequently, these patients require increased vasopressor and fluid substitution because, as in hemorrhagic shock, fast and effective fluid resuscitation is the key to reducing low cardiac output and vasopressor dependency [40-43]. Although the duration of the inflammatory response varies, it is seldom as long as seen in patients experiencing septic shock or sepsis, where it might last for days (not hours) [42-45]. Additionally, HES administration after cardiac surgery may be driven by the lack of therapeutic alternatives. Multiple administrations of crystalloid infusions make the patient prone to fluid overload, which is also a reported risk factor of AKI after cardiac surgery. Furthermore, unnecessary transfusion of blood in patients with sufficient hemoglobin is well known to be associated with adverse outcomes [36, 46-49].

In addition to these physiologic and therapeutic considerations, a prior retrospective exploratory analysis and a prospective randomized controlled trial report similar results in cardiac surgery patients $[50,51]$. In these studies, the administration of HES was considered to be similar to fluid resuscitation with albumin with regard to adverse kidney outcomes [51]. Moreover, an earlier major prospective study concerning fluid management in the ICU also reported a lower mortality rate in patients when initial fluid resuscitation was achieved by the use of colloids (including HES) [52]. Unfortunately, different colloids were used in different study centers, thus no definite conclusions can be drawn regarding the use of HES in particular [52]. Another prospective study in abdominal surgery revealed no significant difference between the administration of crystalloids and colloids. However, the risk for AKI was slightly higher in the HES group [17].

In summary, the existing evidence is conflicting. Different independent studies could not detect an increased risk for adverse outcomes after postoperative administration of HES in surgery patients in the absence of septic shock including cardiac surgery patients [17, 50-54]. Nonetheless, one has to keep in mind that the absence of harm after HES administration in our study does not reflect a benefit or superiority of HES over crystalloids, which needs to be evaluated in prospective randomized controlled trials. These findings however underline that distinct patient populations and settings might need to be taken into account more thoroughly when evaluating adverse renal outcomes after HES administration, rather than generalizing data conclusions about one population and setting for another [55]. With this real-world analysis, we further expand the safety data with respect to administered doses, the dosage timing, and the corresponding impact on renal function.

The study has several limitations. First, we performed a retrospective single-center analysis of an elective patient population. While our patient population was highly restricted to obtain robust conclusions from a homogeneous population, it is of great interest for future studies to examine other populations as well (e.g., mitral valve replacement, aortic valve reconstruction, TAVI, heart transplantation, and assist device implantation). Because fluid management potentially differs between centers and is defined by local guidelines and clinical experience, transferability of the data might be restricted. For example, our data source did not include the information on which surgeon performed the surgery, thus the correlation within the surgeons could not be accounted for. However, this missing information can be considered negligible because no outcome changes have been reported between hospitals and different surgeons [56]. Moreover, we reported the institutional approach to fluid management while strictly defining and accounting for a large number of thoroughly reported patient parameters. Although the limitation of HES use by the European Medicines Agency warning in 2013 affected only patients with septic shock or burns, this notification may nevertheless have influenced (directly or indirectly) the prescribing behavior. Our entire study period covered the period thereafter, thus selection bias during the study was to be ruled out, but the overall situation must be considered interpretatively. Although retrospective studies often have a risk of confounding by indication, the risk in this study appears to be relatively low. The decision to prescribe HES was made directly after surgery within the first $24 \mathrm{~h}$ in the ICU because fluid dependency due to endothelial leakage postpump is highest during this period. This is also reflected in Table S5 in the ESM: 1839 (98\%) of the patients in the HES cohort received HES within $<24 \mathrm{~h}$ after surgery. As a result of the early administration, negative effects would develop after HES was given to stabilize the patient hemodynamically.

A second limitation relates to unmeasured and thus unadjusted confounders, which can never be ruled out. Considering available information, the analysis was fully adjusted and successfully accounted for imbalances between (baseline) variables, which was confirmed by the propensity scoreadjusted sensitivity analysis. We did not consider the latter as the main analysis, as the administration of HES is not binary, but rather a series of complex questions such as dosage, timing, and a combination with crystalloids. Thus, crystalloids and starches had to be operationalized as non-exclusive options, for which covariate adjustment appears most appropriate, but the concurring results based on propensity scores are reassuring. Both approaches can 
effectively control for (measured) confounders. For example, baseline creatinine values differed, as expected because HES is contraindicated in renal impairment and therefore was not prescribed. Additionally, other parameters differed, for example, surgery time and aortic cross-clamp time, with longer times in patients treated with HES. As prolonged surgery and CPB time are associated with hemodynamic instability, this might be an indicator for complicated and insufficient fluid substitution. Furthermore, longer surgical time and higher fluid substitution make the patient prone for AKI, thereby increasing the risk in the other direction [36].

Another limitation relates to the short follow-up period concerning AKI. However, because even mild postoperative AKI is linked to long-lasting adverse effects, it appears reasonable to choose immediate postoperative $\mathrm{AKI}$ as a valid outcome that was defined by the current gold standard, the KDIGO criteria [36, 57]. The longitudinal eGFR trajectory must be interpreted against the backdrop of the short followup, as well. Modeling eGFR is subject to time dependency and delayed effects, especially in the early postoperative period with unstable creatinine measurements. Therefore, the onset of AKI cannot be estimated minutely. However, because other more sensitive biomarkers (e.g., interleukin-18) are not measured in routine care, serum creatinine is still used as a standard in different diagnostic criteria. One must be aware of these inherent limitations, and we consider our eGFR modeling as exploratory evidence adding context to the AKI and mortality models, but not as the primary source of (confirmatory) evidence. Ultimately, it must be considered that drawn conclusions from our models represent a global perspective averaged over all patients. Individual treatment decisions and dosages in particular require an individualized approach including many clinical (prognostic) factors. In this respect, no (individualized) treatment recommendation can be based solely on our results.

\section{Conclusions}

The analysis of real-world data of a large cohort of consecutive postoperative cardiac patients did not show an increased risk for adverse kidney outcomes associated with HES. The time-dependent eGFR models of this analysis suggest that administering low HES doses early after surgery might even delay the steady GFR decrease in patients after cardiac surgery. Generalizing safety signals among strongly different populations (e.g., septic patients or elective cardiac surgery patients) appears questionable in the light of our findings. Although this was a retrospective cohort study, the results indicated that HES might be safely administered to cardiac surgery patients with regard to renal outcomes, especially if it is administered early and dosed appropriately.
Supplementary Information The online version contains supplementary material available at https://doi.org/10.1007/s40264-021-01116-5.

Acknowledgements We thank Prof. Dr. Walter-Emil Haefeli, Mathangi Anusha Chandramouli, M.D., and Dr. Torsten Hoppe-Tichy for critically reading the manuscript and their valuable comments. Andreas D. Meid is funded by the Physician-Scientist Program of the Medical Faculty of Heidelberg University. The funding body did not play any role in the design of the study and the collection, analysis, and interpretation of the data and in writing the manuscript.

Funding Open Access funding enabled and organized by Projekt DEAL.

\section{Declarations}

Funding There was no specific funding for this study.

Conflict of interest The authors declare no conflict of interest.

Ethics approval A positive vote of the responsible ethics committee of the Medical Faculty of Heidelberg University (S-385/2019) was obtained before the start of the study.

Consent to participate Not applicable.

Consent for publication Not applicable.

Availability of data and material Data are available upon reasonable request owing to privacy and ethical restrictions from the authors.

Code availability The code for statistical analysis in $\mathrm{R}$ is available from the corresponding author on reasonable request.

Author contributions BM: conceptualization, data curation, investigation, supervision, writing of the original draft, review, and editing. AM: conceptualization, methodology, formal analysis; writing, review, and editing. JR: data curation, investigation, review, and editing. JS: data curation, review, and editing. MV: conceptualization, review, and editing. MK: conceptualization, review, and editing. MZ: conceptualization, data curation, investigation, supervision, writing, review, and editing. All authors read and approved the final version.

Open Access This article is licensed under a Creative Commons Attribution-NonCommercial 4.0 International License, which permits any non-commercial use, sharing, adaptation, distribution and reproduction in any medium or format, as long as you give appropriate credit to the original author(s) and the source, provide a link to the Creative Commons licence, and indicate if changes were made. The images or other third party material in this article are included in the article's Creative Commons licence, unless indicated otherwise in a credit line to the material. If material is not included in the article's Creative Commons licence and your intended use is not permitted by statutory regulation or exceeds the permitted use, you will need to obtain permission directly from the copyright holder. To view a copy of this licence, visit http://creativecommons.org/licenses/by-nc/4.0/.

\section{References}

1. Protsyk V, Rasmussen BS, Guarracino F, Erb J, Turton E, Ender J. Fluid management in cardiac surgery: results of a survey in 
european cardiac anesthesia departments. J Cardiothorac Vasc Anesth. 2017;31(5):1624-9. https://doi.org/10.1053/j.jvca.2017. 04.017.

2. Shaw A, Raghunathan K. Fluid management in cardiac surgery: colloid or crystalloid? Anesthesiol Clin. 2013;31(2):269-80. https://doi.org/10.1016/j.anclin.2012.12.007.

3. Young R. Perioperative fluid and electrolyte management in cardiac surgery: a review. J Extra Corpor Technol. 2012;44(1):P20-6.

4. Yi JM, Bang JY, Choi B, Cho C, Lee YH, Lee EK, et al. Population-based volume kinetics of crystalloids and colloids in healthy volunteers. Sci Rep. 2019;9(1):18638. https://doi.org/10.1038/ s41598-019-55171-1.

5. Hahn RG. Volume kinetics for infusion fluids. Anesthesiology. 2010;113(2):470-81. https://doi.org/10.1097/ALN.0b013e3181 ded88f.

6. Jacob M, Fellahi JL, Chappell D, Kurz A. The impact of hydroxyethyl starches in cardiac surgery: a meta-analysis. Crit Care. 2014;18(6):656. https://doi.org/10.1186/s13054-014-0656-0.

7. Verheij J, van Lingen A, Beishuizen A, Christiaans HM, de Jong JR, Girbes AR, et al. Cardiac response is greater for colloid than saline fluid loading after cardiac or vascular surgery. Intensive Care Med. 2006;32(7):1030-8. https://doi.org/10.1007/ s00134-006-0195-5.

8. Brunkhorst FM, Engel C, Bloos F, Meier-Hellmann A, Ragaller M, Weiler N, et al. Intensive insulin therapy and pentastarch resuscitation in severe sepsis. N Engl J Med. 2008;358(2):125-39. https://doi.org/10.1056/NEJMoa070716.

9. Perner A, Haase N, Guttormsen AB, Tenhunen J, Klemenzson G, Aneman A, et al. Hydroxyethyl starch 130/0.42 versus ringer's acetate in severe sepsis. N Engl J Med. 2012;367(2):124-34. https://doi.org/10.1056/NEJMoa1204242.

10. Myburgh JA, Finfer S, Bellomo R, Billot L, Cass A, Gattas D, et al. Hydroxyethyl starch or saline for fluid resuscitation in intensive care. N Engl J Med. 2012;367(20):1901-11. https://doi.org/ 10.1056/NEJMoa1209759.

11. European Medicines Agency. EMA/4068/2018. PRAC recommends suspending hydroxyethyl-starch solutions for infusion from the market. https://www.ema.europa.eu/en/documents/press-relea se/prac-recommends-suspending-hydroxyethyl-starch-solutionsinfusion-market_en.pdf. Accessed 24 Apr 2020.

12. Annane D, Fuchs-Buder T, Zoellner C, Kaukonen M, Scheeren TWL. EMA recommendation to suspend HES is hazardous. Lancet. 2018;391(10122):736-8. https://doi.org/10.1016/S01406736(18)30254-X.

13. Chappell D, Jacob M. Hydroxyethyl starch: the importance of being earnest. Scand J Trauma Resusc Emerg Med. 2013;9(21):61. https://doi.org/10.1186/1757-7241-21-61.

14. Haase N, Muller R, Perner A. Debate on HES safety is important, but must be based on facts. Scand J Trauma Resusc Emerg Med. 2013;30(21):66. https://doi.org/10.1186/1757-7241-21-66.

15. Chappell D, Jacob M. Twisting and ignoring facts on hydroxyethyl starch is not very helpful. Scand J Trauma Resusc Emerg Med. 2013;9(21):85. https://doi.org/10.1186/1757-7241-21-85.

16. Wiedermann CJ. Ethical publishing in intensive care medicine: a narrative review. World J Crit Care Med. 2016;5(3):171-9. https:// doi.org/10.5492/wjccm.v5.i3.171.

17. Futier E, Garot M, Godet T, Biais M, Verzilli D, Ouattara A, et al. Effect of hydroxyethyl starch vs saline for volume replacement therapy on death or postoperative complications among high-risk patients undergoing major abdominal surgery: the FLASH randomized clinical trial. JAMA. 2020;323(3):225-36. https://doi.org/ 10.1001/jama.2019.20833.

18. von Elm E, Altman DG, Egger M, Pocock SJ, Gotzsche PC, Vandenbroucke JP, et al. Strengthening the reporting of observational studies in epidemiology (STROBE) statement: guidelines for reporting observational studies. BMJ. 2007;335(7624):806-8. https://doi.org/10.1136/bmj.39335.541782.AD.

19. American Society of Anesthesiologists Task Force on Perioperative Blood Management. Practice guidelines for perioperative blood management: an updated report by the American Society of Anesthesiologists Task Force on Perioperative Blood Management. Anesthesiology. 2015;122(2):241-75. https://doi.org/10. 1097/ALN.0000000000000463.

20. Society of Thoracic Surgeons Blood Conservation Guideline Task Force, Ferraris VA, Brown JR, Despotis GJ, Hammon JW, Reece TB, et al. 2011 update to the Society of Thoracic Surgeons and the Society of Cardiovascular Anesthesiologists blood conservation clinical practice guidelines. Ann Thorac Surg. 2011;91(3):944-82. https://doi.org/10.1016/j.athoracsur. 2010.11.078.

21. Serraino GF, Murphy GJ. Routine use of viscoelastic blood tests for diagnosis and treatment of coagulopathic bleeding in cardiac surgery: updated systematic review and meta-analysis. Br J Anaesth. 2017;118(6):823-33. https://doi.org/10.1093/bja/ aex100.

22. Kidney Disease Improving Global Outcomes (KDIGO) Acute Kidney Injury Work Group. KDIGO clinical practice guideline for acute kidney injury. Kidney Int. 2012;Suppl (2):1-138. https:// doi.org/10.1038/kisup.2012.8.

23. Levey AS, Stevens LA, Schmid CH, Zhang YL, Castro AF 3rd, Feldman HI, et al. A new equation to estimate glomerular filtration rate. Ann Intern Med. 2009;150(9):604-12. https://doi.org/ 10.7326/0003-4819-150-9-200905050-00006.

24. Nashef SA, Roques F, Sharples LD, Nilsson J, Smith C, Goldstone AR, et al. EuroSCORE II. Eur J Cardiothorac Surg. 2012;41(4):734-44. https://doi.org/10.1093/ejcts/ezs043.

25. Naughton CA. Drug-induced nephrotoxicity. Am Fam Phys. 2008;78(6):743-50.

26. Awdishu L, Mehta RL. The 6R's of drug induced nephrotoxicity. BMC Nephrol. 2017;18(1):124. https://doi.org/10.1186/ s12882-017-0536-3.

27. Khan S, Loi V, Rosner MH. Drug-induced kidney injury in the elderly. Drugs Aging. 2017;34(10):729-41. https://doi.org/10. 1007/s40266-017-0484-4.

28. Bates D, Mächler M, Bolker B, Walker S. Fitting linear mixedeffects models using lme4. J Stat Softw. 2015;67(1):48. https:// doi.org/10.18637/jss.v067.i01.

29. Nash JC, Varadhan R. Unifying optimization algorithms to aid software system users: optimx for R. J Stat Softw. 2011;43(9):14. https://doi.org/10.18637/jss.v043.i09.

30. Nash JC. On best practice optimization methods in R. J Stat Softw. 2014;60(2):14. https://doi.org/10.18637/jss.v060.i02.

31. Chen S, Tian L, Cai T, Yu M. A general statistical framework for subgroup identification and comparative treatment scoring. Biometrics. 2017;73(4):1199-209. https://doi.org/10.1111/biom. 12676.

32. Simon N, Friedman JH, Hastie T, Tibshirani R. Regularization paths for Cox's proportional hazards model via coordinate descent. J Stat Softw. 2011;39(5):13. https://doi.org/10.18637/jss. v039.i05.

33. van Buuren $\mathrm{S}$, Groothuis-Oudshoorn K. mice: multivariate imputation by chained equations in R. J Stat Softw. 2011;45(3):67. https://doi.org/10.18637/jss.v045.i03.

34. Friedman JH, Hastie T, Tibshirani R. Regularization paths for generalized linear models via coordinate descent. J Stat Softw. 2010;33(1):22. https://doi.org/10.18637/jss.v033.i01.

35. Kuznetsova A, Brockhoff PB, Christensen RHB. lmertest package: tests in linear mixed effects models. J Stat Softw. 2017;82(13):26. https://doi.org/10.18637/jss.v082.i13.

36. Wang Y, Bellomo R. Cardiac surgery-associated acute kidney injury: risk factors, pathophysiology and treatment. Nat Rev 
Nephrol. 2017;13(11):697-711. https://doi.org/10.1038/nrneph. 2017.119.

37. Hobson CE, Yavas S, Segal MS, Schold JD, Tribble CG, Layon AJ, et al. Acute kidney injury is associated with increased long-term mortality after cardiothoracic surgery. Circulation. 2009;119(18):2444-53. https://doi.org/10.1161/CIRCULATIO NAHA.108.800011.

38. Karkouti K, Wijeysundera DN, Yau TM, Callum JL, Cheng DC, Crowther M, et al. Acute kidney injury after cardiac surgery: focus on modifiable risk factors. Circulation. 2009;119(4):495-502. https://doi.org/10.1161/CIRCULATIONAHA.108.786913.

39. Martin C, Jacob M, Vicaut E, Guidet B, Van Aken H, Kurz A. Effect of waxy maize-derived hydroxyethyl starch 130/0.4 on renal function in surgical patients. Anesthesiology. 2013;118(2):38794. https://doi.org/10.1097/ALN.0b013e31827e5569.

40. Paparella D, Yau TM, Young E. Cardiopulmonary bypass induced inflammation: pathophysiology and treatment: an update. Eur J Cardiothorac Surg. 2002;21(2):232-44. https://doi.org/10.1016/ s1010-7940(01)01099-5.

41. MacCallum NS, Finney SJ, Gordon SE, Quinlan GJ, Evans TW. Modified criteria for the systemic inflammatory response syndrome improves their utility following cardiac surgery. Chest. 2014;145(6):1197-203. https://doi.org/10.1378/chest.13-1023.

42. Cremer J, Martin M, Redl H, Bahrami S, Abraham C, Graeter $\mathrm{T}$, et al. Systemic inflammatory response syndrome after cardiac operations. Ann Thorac Surg. 1996;61(6):1714-20. https://doi. org/10.1016/0003-4975(96)00055-0.

43. Squiccimarro E, Labriola C, Malvindi PG, Margari V, Guida P, Visicchio $G$, et al. Prevalence and clinical impact of systemic inflammatory reaction after cardiac surgery. J Cardiothorac Vasc Anesth. 2019;33(6):1682-90. https://doi.org/10.1053/j.jvca.2019. 01.043 .

44. Chuma M, Makishima M, Imai T, Tochikura N, Sakaue T, Kikuchi $\mathrm{N}$, et al. Duration of systemic inflammatory response syndrome influences serum vancomycin concentration in patients with sepsis. Clin Ther. 2016;38(12):2598-609. https://doi.org/10.1016/j. clinthera.2016.10.009.

45. Sugita H, Kinoshita Y, Baba $\mathrm{H}$. The duration of SIRS before organ failure is a significant prognostic factor of sepsis. Int J Emerg Med. 2012;5(1):44. https://doi.org/10.1186/1865-1380-5-44.

46. Gupta PB, DeMario VM, Amin RM, Gehrie EA, Goel R, Lee $\mathrm{KHK}$, et al. Patient blood management program improves blood use and clinical outcomes in orthopedic surgery. Anesthesiology. 2018;129(6):1082-91. https://doi.org/10.1097/ALN.0000000000 002397.

47. Meybohm P, Herrmann E, Steinbicker AU, Wittmann M, Gruenewald M, Fischer D, et al. Patient blood management is associated with a substantial reduction of red blood cell utilization and safe for patient's outcome: a prospective, multicenter cohort study with a noninferiority design. Ann Surg. 2016;264(2):203-11. https://doi.org/10.1097/SLA.0000000000001747.

48. Shander A, Goodnough LT. Why an alternative to blood transfusion? Crit Care Clin. 2009;25(2):261-77. https://doi.org/10. 1016/j.ccc.2008.12.012.

49. Haase-Fielitz A, Haase M, Bellomo R, Calzavacca P, Spura $\mathrm{A}$, Baraki $\mathrm{H}$, et al. Perioperative hemodynamic instability and fluid overload are associated with increasing acute kidney injury severity and worse outcome after cardiac surgery. Blood Purif. 2017;43(4):298-308. https://doi.org/10.1159/000455061.

50. Momeni M, Nkoy Ena L, Van Dyck M, Matta A, Kahn D, Thiry D, et al. The dose of hydroxyethyl starch $6 \% 130 / 0.4$ for fluid therapy and the incidence of acute kidney injury after cardiac surgery: a retrospective matched study. PLoS ONE. 2017;12(10):186403. https://doi.org/10.1371/journal.pone.0186403.

51. Duncan AE, Jia Y, Soltesz E, Leung S, Yilmaz HO, Mao G, et al. Effect of $6 \%$ hydroxyethyl starch $130 / 0.4$ on kidney and haemostatic function in cardiac surgical patients: a randomised controlled trial. Anaesthesia. 2020;75(9):1180-90. https://doi.org/ 10.1111/anae.14994.

52. Annane D, Siami S, Jaber S, Martin C, Elatrous S, Declere $\mathrm{AD}$, et al. Effects of fluid resuscitation with colloids vs crystalloids on mortality in critically ill patients presenting with hypovolemic shock: the CRISTAL randomized trial. JAMA. 2013;310(17):1809-17. https://doi.org/10.1001/jama.2013. 280502.

53. Gillies MA, Habicher M, Jhanji S, Sander M, Mythen M, Hamilton $\mathrm{M}$, et al. Incidence of postoperative death and acute kidney injury associated with iv $6 \%$ hydroxyethyl starch use: systematic review and meta-analysis. Br J Anaesth. 2014;112(1):25-34. https://doi.org/10.1093/bja/aet303.

54. Raiman M, Mitchell CG, Biccard BM, Rodseth RN. Comparison of hydroxyethyl starch colloids with crystalloids for surgical patients: a systematic review and meta-analysis. Eur J Anaesthesiol. 2016;33(1):42-8. https://doi.org/10.1097/EJA.0000000000 000328.

55. Crowe B, Chuang-Stein C, Lettis S, Brueckner A. Reporting adverse drug reactions in product labels. Ther Innov Regul Sci. 2016;50(4):455-63. https://doi.org/10.1177/2168479016628574.

56. Soppa G, Theodoropoulos P, Bilkhu R, Harrison DA, Alam $\mathrm{R}$, Beattie R, et al. Variation between hospitals in outcomes following cardiac surgery in the UK. Ann R Coll Surg Engl. 2019;101(5):333-41. https://doi.org/10.1308/rcsann.2019.0029.

57. Turan A, Cohen B, Adegboye J, Makarova N, Liu L, Mascha EJ, et al. Mild acute kidney injury after noncardiac surgery is associated with long-term renal dysfunction: a retrospective cohort study. Anesthesiology. 2020;132(5):1053-61. https://doi.org/10. 1097/ALN.0000000000003109. 